\title{
Is Assessment of Femoral Head Perfusion During Modified Dunn for Unstable Slipped Capital Femoral Epiphysis an Accurate Indicator of Osteonecrosis?
}

\author{
Eduardo N. Novais MD, Ernest L. Sink MD, Lauryn A. Kestel BS, \\ Patrick M. Carry BA, João C. M. Abdo MD, Travis C. Heare MD
}

Received: 10 December 2015/Accepted: 30 March 2016/Published online: 18 April 2016

(C) The Association of Bone and Joint Surgeons (B) 2016

\begin{abstract}
Background The modified Dunn procedure, which is an open subcapital realignment through a surgical dislocation approach, has gained popularity for the treatment of unstable slipped capital femoral epiphysis (SCFE). Intraoperative monitoring of the femoral head perfusion has been recommended as a method of predicting osteonecrosis; however, the accuracy of this assessment has not been well documented.

Questions/purposes We asked (1) whether intraoperative assessment of femoral head perfusion would help identify

Each author certifies that he or she, or a member of his or her immediate family, has no funding or commercial associations (eg, consultancies, stock ownership, equity interest, patent/licensing arrangements, etc) that might pose a conflict of interest in connection with the submitted article.

All ICMJE Conflict of Interest Forms for authors and Clinical Orthopaedics and Related Research ${ }^{\mathbb{R}}$ editors and board members are on file with the publication and can be viewed on request.

Clinical Orthopaedics and Related Research ${ }^{\circledR}$ neither advocates nor endorses the use of any treatment, drug, or device. Readers are encouraged to always seek additional information, including FDAapproval status, of any drug or device prior to clinical use.

Each author certifies that his or her institution approved the human protocol for this investigation, that all investigations were conducted in conformity with ethical principles of research, and that informed consent for participation in the study was obtained.

This work was performed at Children's Hospital Colorado, Aurora, CO, USA.
\end{abstract}

E. N. Novais ( $\square)$

Department of Orthopaedic Surgery, Boston Children's Hospital,

300 Longwood Avenue, Hunnewell Building, Boston, MA

02215 , USA

e-mail: eduardo.novais@childrens.harvard.edu

E. L. Sink

Hip Center at Hospital for Special Surgery, New York, NY, USA hips at risk of developing osteonecrosis; (2) whether one of the four methods of assessment of femoral head perfusion is more accurate (highest area under the curve) at identifying hips at risk of osteonecrosis; and (3) whether specific clinical features would be associated with osteonecrosis occurrence after a modified Dunn procedure for unstable SCFE.

Methods Between 2007 and 2014, we performed 29 modified Dunn procedures for unstable SCFE (16 boys, 11 girls; median age, 13 years; range, $8-17$ years); two were lost to followup before 1 year. During this period, six patients with unstable SCFE were treated by other procedures. All patients undergoing modified Dunn underwent assessment of epiphyseal perfusion by the presence of active bleeding and/or by intracranial pressure (ICP) monitoring. In the initial five patients perfusion was recorded once, either before dissection of the retinacular flap or after fixation by one of the two methods. In the remaining 22 patients $(81 \%)$, perfusion was systematically assessed before dissection of the retinacular flap and after fixation by both methods. Minimum followup was 1 year (median, 2.5 years; range, 18 years) because osteonecrosis typically develops within the first year after surgery. Patients were assessed for osteonecrosis by the presence of femoral head collapse at radiographs obtained every 3 months during the first year after surgery. Seven $(26 \%)$ of the 27 patients developed

L. A. Kestel, P. M. Carry, T. C. Heare

Department of Orthopaedic Surgery, Children's Hospital

Colorado, Aurora, CO, USA

J. C. M. Abdo

Hospital Pequeno Principe, Curitiba, Brazil 
osteonecrosis. Measures of diagnostic accuracy including sensitivity, specificity, and the area under the receiver operating curve (AUC) were estimated. Multiple variable logistic regression analyses were used to test whether the test options were better than random chance (AUC $>0.50$ ) at differentiating between patients who did versus did not develop osteonecrosis. Nonparametric methods were used to test for a difference in AUC across the four methods. A secondary analysis was performed to identify risk factors associated with osteonecrosis.

Results After adjusting for body mass index, which was found to be a confounding variable, assessment of femoral head perfusion with ICP monitoring before retinaculum dissection (adjusted AUC: 0.79; 95\% confidence interval $[C I], 0.58-0.99 ; p=0.006$ ), femoral head perfusion with ICP monitoring after definitive fixation (adjusted AUC: 0.82 ; 95\% CI, $0.65-1.0 ; \mathrm{p}<0.001$ ), bleeding before retinaculum dissection (adjusted AUC: 0.77; 95\% CI, 0.58 $0.96 ; \mathrm{p}=0.006$ ), and bleeding after definitive fixation (adjusted AUC: 0.81; 95\% CI, 0.63-0.99; p = 0.001) were found to be helpful at identifying osteonecrosis. We were not able to identify a specific test that had performed best because there was no difference $(p=0.8226)$ in AUC across the four methods. With the numbers available, we were unable to identify clinical factors predictive of osteonecrosis in our cohort.

Conclusions Assessments of femoral head blood perfusion by ICP monitoring or by the presence of active bleeding in combination with the patient's body mass index are effective at differentiating between patients who do versus do not develop osteonecrosis after a modified Dunn procedure for unstable SCFE. Additional research is needed to determine whether information gained from assessment of femoral head perfusion during surgery should be used to guide targeted treatment recommendations that may reduce the development of femoral head deformity secondary to osteonecrosis.

Level of Evidence Level III, diagnostic study.

\section{Introduction}

Slipped capital femoral epiphysis (SCFE) is classified as unstable when the patient reports a level of pain that, regardless of the duration of the symptoms, is severe enough to prevent walking even with crutches [16]. Instability of the SCFE has been cited as an important prognostic factor as a result of its association with the development of osteonecrosis of the femoral head [16, 17, 27-29]. Osteonecrosis is the most severe complication after unstable SCFE treatment. Although previous studies [10, $16,17,19,20,23,27-29]$ vary widely in terms of the proportion of patients who develop osteonecrosis after unstable SCFE, a recent systematic review of the literature reported an overall rate of $24 \%$ [29]. Osteonecrosis leads to femoral head collapse and further hip osteoarthritis, and it is the most common indication for hip arthroplasty in patients with SCFE [11, 12].

Several surgical techniques including in situ pinning $[19,27]$, reduction with a decompression arthrotomy and pinning [1, 6], and open reduction [20] have been described for the treatment of unstable SCFE. The recently described surgical dislocation approach [4] with dissection of the retinacular flap containing the nutrient vessels to the femoral head [5] has allowed for advances in the surgical treatment of SCFE using a modification of the Dunn [3] subcapital realignment procedure, in which open realignment of the femoral epiphysis is performed. One of the benefits of the subcapital realignment through the surgical dislocation approach (modified Dunn procedure) is visualization and protection of the vascular retinaculum and direct assessment of perfusion of the femoral head during surgery. Femoral head perfusion may be assessed by evaluating bleeding after creating a $2-\mathrm{mm}$ drill hole $[9,24$, $30,31]$, by laser-Doppler flowmetry [9, 30], and/or by intracranial pressure probe monitoring [24]. Assessment of perfusion during surgery may allow for adjustments of positioning the femoral head if blood flow decreases after the femoral head is reduced. In addition, it may allow for identification of patients at risk of developing osteonecrosis long before femoral head deformity develops, which would allow for potential early interventions to prevent such deformities. Although commonly performed, the accuracy of femoral head perfusion assessment during modified Dunn in unstable SCFE has not been well documented.

In this retrospective study we asked (1) whether intraoperative assessment of femoral head perfusion would help identify hips at risk of developing osteonecrosis; (2) whether one of the four methods of assessment of femoral head perfusion is more accurate (highest area under the curve) at identifying hips at risk of osteonecrosis; and (3) whether specific clinical features would be associated with osteonecrosis occurrence after a modified Dunn procedure for unstable SCFE.

\section{Patients and Methods}

In March 2007 we began performing the modified Dunn procedure for treatment of patients with an unstable SCFE as defined by the Loder [15]. Since 2011, we treated all patients with unstable SCFE with the modified Dunn procedure. Between March 2007 and 2014, we performed 29 modified Dunn procedures for unstable SCFE in 16 boys and 11 girls with a median age of 13 (range, 8-17 years). During the study period, six patients were treated by in situ 
pinning based on the indication by the orthopaedic surgeon on-call. Two patients who were lost to followup before 1 year were excluded from the study, leaving 27 patients followed for a minimum of 1 year. Because osteonecrosis after unstable SCFE typically develops within the first year of surgery [15, 16, 24], we set minimum followup of this study to 1 year. The median duration of followup was 2.5 years (range, 1-8 years).

One of the authors (LAK) retrospectively reviewed the medical records for collection of preoperative variables including age at surgery, sex, body mass index, estimated time from injury to surgery, and SCFE chronicity. Severity of SCFE was assessed on AP pelvic preoperative radiographs and on frog-leg view radiographs when available [26]. Intraoperative notes were reviewed for the purpose of assessing femoral head bleeding and damage to the periosteum and retinacular vessels and determining the type of fixation used.

Surgical treatment was performed by one of the three senior authors (ENN, TCH, ELS) following a previously described technique $[13,14,31]$. Ten of the 27 patients (37\%) were operated less than 24 hours after the slip, whereas 17 of $27(63 \%)$ were operated on 24 hours after the slip. Briefly, the patient was positioned in lateral decubitus and a straight lateral incision was performed. The fascia lata is incised anteriorly to the gluteus maximus to avoid splitting the muscle. A trochanteric osteotomy was performed and the hip capsule was exposed. After capsulotomy, the metaphysis of the proximal femur was exposed and two-threaded Kirschner wires were used to provisionally stabilize the epiphysis. Before dissection of the retinacular flap, femoral head perfusion was assessed by drilling a 2-mm hole in the anterosuperior aspect. The presence or absence of active bleeding was recorded (Fig. 1). An Integra ${ }^{\circledR}$ Camino $^{\circledR}$ Intracranial Pressure

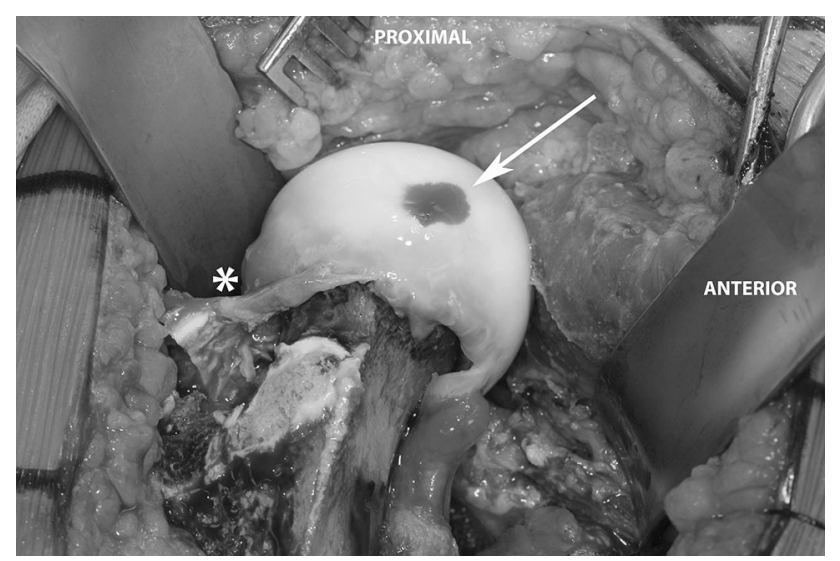

Fig. 1 Intraoperative picture during a modified Dunn procedure after definitive fixation of the epiphysis shows the presences of active bleeding through a 2-mm drill hole (arrow) and the intact vascular retinaculum $(*)$.
Monitoring (Integra, Plainsboro, NJ, USA) display was adjusted to read zero and the intracranial pressure (ICP) probe was inserted into the drill hole. The scale of pressure waveform on the display was adjusted to the range of -5 $\mathrm{mmHg}$ to $10 \mathrm{mmHg}$. Although the intracranial pressure (ICP) monitor displays a numeric value of pressure, the waveform was recorded as present when there was a large waveform with a clear peak. The ICP monitor reading was interpreted as negative when there was a flat line with no clear waveform on display or in patients with very minimal wave changes on the display.

The hip was then dislocated after release of the teres ligament by flexion and external rotation. The acetabular cartilage and labrum were assessed and the hip was reduced. An osteotomy was performed at the physis of the greater trochanter for subperiosteal dissection of the retinacular flap [5]. The retinaculum was dissected all the way posteriorly and after the hip was again dislocated and the femoral neck was subperiosteally exposed anteriorly and medially. Posterior and inferior callus was resected; however, formal femoral neck shortening was avoided. After the epiphysis was curetted to remove the physeal cartilage, the epiphysis was reduced carefully into the femoral neck and the alignment and tension to the retinaculum were checked. The epiphysis was then provisionally fixed with two threaded Kirschner wires or with the guidewires for a cannulated screw. The femoral head was reduced in the acetabulum and the epiphyseal bleeding was reassessed. In cases in which the perfusion of the femoral head was found to be absent or reduced, the tension on the retinaculum was checked and if necessary the femoral neck was shortened slightly not to exceed $3 \mathrm{~mm}$. Radiographic alignment of the epiphysis was confirmed and final fixation was obtained with three threaded Kirschner wires or two cannulated 6.5$\mathrm{mm}$ screws, according to surgeon preference. Final femoral head active bleeding and ICP monitor waveform were recorded with the hip in neutral position of abduction and rotation. The capsule was loosely closed. The trochanteric fragment was fixed with either two or three $3.5-\mathrm{mm}$ or 4.5$\mathrm{mm}$ screws using a standard compression technique. The wound was closed in layers. Patients were advised to avoid bearing weight and allowed to use the contralateral leg for pivoting transfer in a wheelchair for minimum of 6 weeks. After this period, when radiographic healing was observed, patients were instructed to follow physical therapy-guided rehabilitation to achieve full bearing of weight.

One of the authors (JCMA) assessed postoperative radiographs for the presence of osteonecrosis of the femoral head. Osteonecrosis was defined as observations of changes in the femoral head including progressive sclerosis followed by cystic changes and collapse. Patients were typically evaluated clinically and with an AP and frog-leg radiograph at 6 and 12 weeks after surgery and then at 6 
months, 9 months, and at minimum 12 months postoperatively. Seven of the 27 patients (26\%; exact 95\% confidence interval, $11 \%-46 \%$ ) developed osteonecrosis of the femoral head. The diagnosis was made at a median of 3 months after surgery (range, 2-16 months). All patients who developed osteonecrosis were diagnosed during the first 6 months after surgery with the exception of one patient. A 12-year-old girl was seen at the 3-month followup and was instructed to return for the 6-month postoperative visit. However, as a result of social issues, the patient was not seen until 409 days after surgery when she was seen with a major limp, pain, hip contracture, and complete resorption of the femoral head on radiographs. Among the seven patients who developed osteonecrosis, two had a complete tear of the retinaculum with nearly full avulsion from the epiphysis observed during surgery. These patients had absent perfusion of the femoral head throughout the entire procedure.

All patients undergoing the modified Dunn procedure underwent assessment of epiphyseal perfusion by the presence of active bleeding and/or by ICP probe monitoring. Given the retrospective nature of the study, in the initial five patients in this series, perfusion was recorded once, either before dissection of the retinacular flap or after fixation by one of the two methods. In the remaining 22 patients $(81 \%)$, perfusion was systematically assessed before dissection of the retinacular flap and after fixation by both methods.

For the purpose of this study, femoral head perfusion was defined by the presence (negative test) or absence (positive test) of a waveform on the ICP monitor display and the presence (negative test) or absence (positive test) of active bleeding before the subperiosteal dissection of the retinaculum and after definitive fixation of the epiphysis. Measures of diagnostic accuracy including sensitivity, specificity, and the area under the receiver operating curve (AUC) along with their corresponding 95\% confidence intervals were estimated for each method of detecting osteonecrosis. Among patients who truly develop osteonecrosis, sensitivity represents the probability a given test is positive. Among patients who truly do not develop osteonecrosis, specificity represents the probability a given test is negative. A receiver operating curve (ROC curve) is constructed graphically by plotting the sensitivity on the Yaxis and 1-specificity on the $\mathrm{X}$ axis. The area under the curve represents the total area under the ROC curve and describes the accuracy of a test. Values range between 0 and 1 with higher values indicative of better test performance. Values of 0.5 suggest the test is equivalent to random chance at differentiating between patients who do versus do not develop osteonecrosis. Generally, AUC values can be interpreted as the probability that among a given pair of patients (one with osteonecrosis and one without), the test will correctly identify the patient with osteonecrosis [7]. When comparing test options, the test associated with the higher AUC value is generally considered a superior test [22].

Multivariable logistic regression models (Proc Logistic; SAS 9.4, Cary, NC, USA) were used to test the null hypothesis that the AUC values obtained from the models representing each of the four methods of assessing perfusion status were $\leq 0.50$. The nonparametric methods described by DeLong et al. [2] were used to test for difference in AUC across the four methods. Based on the distribution of body mass index (BMI) across subjects who did versus did not develop osteonecrosis, all models included BMI as a cofounding variable. A secondary analysis investigated potential factors associated with osteonecrosis. Descriptive statistics were used to summarize the demographics and clinical characteristics of subjects who did versus did not develop osteonecrosis after the surgical management of an unstable SCFE. Wilcoxon rank-sum or Fisher's exact test was used to compare the distribution of these variables across the two osteonecrosis groups.

\section{Results}

After adjusting for BMI, the assessment of femoral head perfusion with ICP monitoring before retinaculum dissection (adjusted AUC: 0.79; 95\% confidence interval [CI], $0.58-0.99 ; \mathrm{p}=0.006$ ), the assessment of femoral head perfusion with ICP monitoring after definitive fixation (adjusted AUC: $0.82 ; 95 \%$ CI, 0.65-1.0; $\mathrm{p}<0.001$ ), the presence of femoral head bleeding before retinaculum dissection (adjusted AUC: 0.77; 95\% CI, 0.58-0.96; $\mathrm{p}=$ 0.006), and the presence of femoral head bleeding after definitive fixation (adjusted AUC: 0.81 ; 95\% CI, 0.63$0.99 ; p=0.001$ ) were superior to random chance at differentiating between patients who did versus did not develop osteonecrosis during the study period.

There was no difference in AUC across the four methods of assessing femoral head perfusion status $(p=0.8226)$. A descriptive review of the unadjusted measures of diagnostic accuracy revealed that assessment of active bleeding after fixation had excellent $(1.0 ; 95 \%$ CI, 0.82-1.0) specificity, whereas ICP monitoring before retinaculum dissection had good sensitivity $(0.67 ; 95 \%$ CI, $0.22-0.96$; Table 1).

With the numbers available, we found no clinical features that allowed us to predict which hips were at risk of developing osteonecrosis. The mean BMI of patients who did not progress to osteonecrosis was $25 \mathrm{~kg} / \mathrm{m}^{2}$ (SD 6), whereas the mean BMI of patients who did was $30 \mathrm{~kg} / \mathrm{m}^{2}$ $(\mathrm{SD} 5)(\mathrm{p}=0.0818)($ Table 2). 
Table 1. Unadjusted measures of diagnostic accuracy associated with the four methods of assessing femoral head perfusion

\begin{tabular}{|c|c|c|c|c|c|c|c|}
\hline Method of perfusion assessment & $\begin{array}{l}\text { True- } \\
\text { positive }\end{array}$ & $\begin{array}{l}\text { False- } \\
\text { positive }\end{array}$ & $\begin{array}{l}\text { True- } \\
\text { negative }\end{array}$ & $\begin{array}{l}\text { False- } \\
\text { negative }\end{array}$ & $\begin{array}{l}\text { Sensitivity } \\
\left(95 \% \mathrm{CI}^{*}\right)\end{array}$ & $\begin{array}{l}\text { Specificity } \\
\left(95 \% \mathrm{CI}^{*}\right)\end{array}$ & $\begin{array}{l}\text { Area under } \\
\text { the curve } \\
(95 \% \mathrm{CI})\end{array}$ \\
\hline $\begin{array}{l}\text { ICP monitoring before retinacular } \\
\text { dissection } \\
\text { (+ no waveform; - waveform } \\
\text { present })\end{array}$ & 4 & 7 & 9 & 2 & $0.67(0.22-0.96)$ & $0.56(0.30-0.80)$ & $0.61(0.37-0.86)$ \\
\hline $\begin{array}{l}\text { ICP monitoring after definitive } \\
\text { fixation } \\
\text { (+ no waveform; - waveform } \\
\text { present) }\end{array}$ & 3 & 3 & 14 & 3 & $0.50(0.12-0.88)$ & $0.82(0.57-0.96)$ & $0.66(0.42-0.90)$ \\
\hline $\begin{array}{l}\text { Active bleeding before retinacular } \\
\text { dissection } \\
(+ \text { absent bleeding; }- \text { bleeding } \\
\text { present })\end{array}$ & 3 & 5 & 14 & 4 & $0.43(0.10-0.82)$ & $0.74(0.49-0.91)$ & $0.58(0.36-0.81)$ \\
\hline $\begin{array}{l}\text { Active bleeding after definitive } \\
\text { fixation } \\
\text { (+ absent bleeding; - bleeding } \\
\text { present) }\end{array}$ & 1 & 0 & 19 & 6 & $0.14(0.00-0.58)$ & $1.00(0.82-1.00)$ & $0.57(0.43-0.71)$ \\
\hline
\end{tabular}

* Exact 95\% binomial confidence interval; true-positive = positive test and osteonecrosis present; false-positive = positive test and osteonecrosis absent; true-negative $=$ negative test and osteonecrosis present; false-negative $=$ positive test and osteonecrosis absent.

Table 2. Comparison of patients who did versus did not develop osteonecrosis of the femoral head

\begin{tabular}{|c|c|c|c|c|c|}
\hline \multirow{2}{*}{$\begin{array}{l}\text { Patient, SCFE, and surgical } \\
\text { characteristics }\end{array}$} & \multicolumn{2}{|c|}{ No osteonecrosis } & \multicolumn{2}{|c|}{ Osteonecrosis } & \multirow[t]{2}{*}{$\mathrm{p}$ value } \\
\hline & \multicolumn{2}{|c|}{$(\mathrm{N}=20)$} & \multicolumn{2}{|c|}{$(\mathrm{N}=7)$} & \\
\hline \multicolumn{6}{|l|}{ Time to surgery } \\
\hline$\leq 24$ hours & 9 & $90 \%$ & 1 & $10 \%$ & 0.204 \\
\hline$>24$ hours & 11 & $65 \%$ & 6 & $35 \%$ & \\
\hline \multicolumn{6}{|l|}{ Type of fixation } \\
\hline Cannulated screws & 10 & $77 \%$ & 3 & $23 \%$ & $>0.999$ \\
\hline Pins & 10 & $71 \%$ & 4 & $29 \%$ & \\
\hline \multicolumn{6}{|l|}{ Grade } \\
\hline Moderate & 2 & $100 \%$ & 0 & $0 \%$ & $>0.999$ \\
\hline Severe & 18 & $72 \%$ & 7 & $6 \%$ & \\
\hline \multicolumn{6}{|l|}{ Pain } \\
\hline$\leq 1$ week & 11 & $79 \%$ & 3 & $21 \%$ & 0.665 \\
\hline$>1$ week & 8 & $67 \%$ & 4 & $33 \%$ & \\
\hline Age* & 12.5 & Range, 7-17 & 13 & Range, 11-15 & 0.604 \\
\hline BMI* & 24.6 & Range, 16-37 & 29 & Range, 24-38 & 0.068 \\
\hline
\end{tabular}

* BMI was included as a cofounding variable in the multivariate analysis; SCFE = slipped capital femoral epiphysis; BMI = body mass index.

\section{Discussion}

There has been a recent increase in interest of the modified Dunn procedure for the treatment of SCFE [24]. The increased attention is most likely related to the fact that osteonecrosis of the femoral head was not reported in the first descriptive studies [13, 14, 29]. Recent studies, however, have reported that approximately $25 \%$ of patients undergoing a modified Dunn for unstable SCFE developed osteonecrosis [21, 22]. One of the advantages of the modified Dunn procedure is the ability to perform an intraoperative assessment of epiphyseal perfusion and controlled reduction of the epiphysis preserving its blood supply. However, there is limited evidence [18, 30] about the performance of epiphyseal perfusion assessment in screening for osteonecrosis. In this study, we investigated the accuracy of intraoperative assessment of femoral head vascularity by examining the presence of active bleeding 
and using an ICP monitor as an indicator for osteonecrosis development in patients undergoing a modified Dunn procedure for the treatment of unstable SCFE while assessing clinical features as possible prognostic factors. We found that after adjusting for BMI, all four methods of assessing femoral head perfusion were superior to random chance at differentiating between patients who did versus did not have osteonecrosis by the end of the study period.

This study has several limitations. First, this was a retrospective study based on data collected from operative notes over a relatively long time. In the initial five patients in this series, perfusion was recorded once, either before dissection of the retinacular flap or after fixation by one of the two methods. In the remaining 22 patients $(81 \%)$, perfusion was systematically assessed before dissection of the retinacular flap and after fixation by both methods. Although this may have limited the number of patients with complete data, we do not believe that this compromises our findings because it reflects our evolution in assessing femoral head perfusion rather than a selective assessment. Second, our cohort represents the learning curve of the three surgeons. Thus, the overall proportion of patients who developed osteonecrosis may be higher than the expected proportion reported by the originators of the technique [13, 14, 29]. However, all surgeons were experienced in the surgical dislocation approach and the proportion of osteonecrosis in this series compares well to other recent studies [24, 25]. We believe that by including multiple surgeons with relevant expertise, we improved the external validity of our study. Third, the assessment of femoral head perfusion using the presence of active bleeding and ICP monitoring may be subjective. In agreement with a previous study [6], we noted that during surgery, the quality of the femoral head bleeding after drilling was not necessarily homogeneous. In some patients, immediate bright red blood came out of the hole, whereas in others, the bleeding was darker and more delayed. For the ICP monitoring, we did not use an objective assessment of the actual numeric value of the pressure because the device was not developed for intraosseous pressure monitoring. We collected the presence of an ample waveform versus an absent waveform. The few patients in whom minimal changes in the monitor display were seen were classified as having no waveform present. Fourth, during the first 4 years of the study, six patients with unstable SCFE were treated by other methods, which could raise a concern for selection bias. However, the indication for treatment on these cases was solely based on the treating surgeon who was on call rather than on severity of the SCFE. Since 2011 all patients with unstable SCFE have been treated by the modified Dunn approach. Fifth, as a result of the small number of patients, our study was limited in power to find factors predictive of osteonecrosis. However, unstable SCFE is an uncommon condition and larger investigations involving multiple centers are needed to accumulate a large number of patients. Finally, the followup on this series of patients is relatively short, and because the series is small, it would not take very many events in the test $(+)$ group or the test (-) group for our conclusions to change, which reflects low statistical power. However, our experience as well as that of other investigators $[15,16,24]$ is that the development of osteonecrosis after SCFE occurs within the first 12 months of treatment. In this series, all patients who were diagnosed with osteonecrosis developed it within a year of treatment.

We found that in combination with patients' characteristics, namely BMI, the four methods of assessing femoral head perfusion were effective at differentiating between patients who do versus do not develop osteonecrosis after a modified Dunn procedure for unstable SCFE. The information gained from assessing femoral head perfusion may be used to help the surgeon during and after the procedure. For instance, if the femoral head is found to have perfusion before dissection of the retinaculum, it is expected that perfusion will be maintained throughout the procedure. If perfusion is lost after reduction and fixation, the surgeon may be able to adjust the head realignment and position, potentially shorten the femoral neck slightly, or revisit the flap dissection to reestablish the blood flow. Another potential benefit of intraoperative assessment of perfusion is identification of patients at risk of developing osteonecrosis before radiographic changes take place, which may allow for early interventions to prevent severe deformity secondary to epiphyseal collapse. Complete absence of epiphyseal perfusion (before dissection and after fixation) was highly indicative of osteonecrosis and this may be an indication for further treatment including intravenous bisphosphonates [21].

Our data did not favor one test over the other for assessment of femoral head perfusion during modified Dunn for unstable SCFE. However, a descriptive analysis of the raw measurements of accuracy showed that ICP monitoring before dissection demonstrated good sensitivity, whereas bleeding after definitive fixation was a highly (100\%) specific test. The absence of femoral head perfusion after fixation was also found to have high specificity by Madan et al. [18] who reported osteonecrosis in four our of 17 patients with unstable SCFE who had no bleeding before dislocation and after reduction. Similarly, Sankar et al. [24] found no perfusion after fixation in three of 27 unstable SCFEs. All three patients developed osteonecrosis. However, in accordance with Ziebarth et al. [30], we observed two patients with an intact retinacular flap in whom there was no intraoperative ICP waveform and who did not development of osteonecrosis. These represent examples of a false-positive test result. It is unclear, however, whether 
revascularization was related to the integrity of the retinacular vessels or if it may have happened through a transphyseal mechanism. Contrary to previous studies [9, $18,30]$, we found three patients with an ICP waveform before dissection and after fixation who later developed osteonecrosis. These represent examples of a false-negative test result. In two patients, the femoral head was well perfused at baseline and it is possible that osteonecrosis developed in association with tension of the capsule closure. This, however, was also reported by Sankar et al. [24] who found four patients who developed osteonecrosis despite confirmed blood flow after fixation. Continuing with monitoring toward the end of the procedure, although technically challenging, may allow for identification of cases that lose blood supply after closure of the capsule.

With the numbers available, we were not able to identify any factors related to osteonecrosis of the femoral head after a modified Dunn procedure for unstable SCFE. Similar to Sankar et al. [24], we did not identify timing to surgery as a factor. This may be related to the fact that we did not perform any surgery within 8 hours after presentation. In contrast, Ziebarth et al. [30] showed that patients undergoing a modified Dunn procedure less than 8 hours from injury were less likely to develop osteonecrosis when compared with patients undergoing surgery after 8 hours. Early surgery may have the benefit of decompression of the hematoma, which has been associated with increased intraarticular pressure, a potential risk factor for osteonecrosis [8]. Although the etiology of osteonecrosis after unstable SCFE remains controversial, our data confirmed traumatic rupture of the retinaculum as an unusual mechanism.

The modified Dunn procedure allows for controlled visualization and protection of the vascular retinaculum and assessment of the blood flow to the femoral head during surgery. Our findings suggested that assessment of femoral head active bleeding or ICP monitoring is effective in identifying patients who are at high risk of developing osteonecrosis when they are used in combination with patient specific factors such as BMI. In practice the treating surgeon should carefully consider whether it is preferable to rely on a test with high sensitivity (ICP monitoring before retinacular dissection) to minimize the number of false-negatives or if it is preferable to choose a test with high specificity (assessment of active bleeding after fixation) to minimize the number of false-positives and overtreatment. Because osteonecrosis is a severe complication of unstable SCFE with a high risk of progressive hip degenerative osteoarthritis [12], we believe that use of a test with high sensitivity is preferable, because it would include all patients who would develop osteonecrosis. In our study, total lack of blood flow before dissection and after fixation was highly indicative of risk of necrosis. This finding may thus be an indication for further intervention.

\section{References}

1. Chen RC, Schoenecker PL, Dobbs MB, Luhmann SJ, Szymanski DA, Gordon JE. Urgent reduction, fixation, and arthrotomy for unstable slipped capital femoral epiphysis. J Pediatr Orthop. 2009;29:687-694.

2. DeLong ER, DeLong DM, Clarke-Pearson DL. Comparing the areas under two or more correlated receiver operating characteristic curves: a nonparametric approach. Biometrics. 1988;44:837-845.

3. Dunn DM. The treatment of adolescent slipping of the upper femoral epiphysis. J Bone Joint Surg Br. 1964;46:621-629.

4. Ganz R, Gill TJ, Gautier E, Ganz K, Krugel N, Berlemann U. Surgical dislocation of the adult hip a technique with full access to the femoral head and acetabulum without the risk of avascular necrosis. J Bone Joint Surg Br. 2001;83:1119-1124.

5. Ganz R, Huff TW, Leunig M. Extended retinacular soft-tissue flap for intra-articular hip surgery: surgical technique, indications, and results of application. Instr Course Lect. 2009;58:241-255.

6. Gordon JE, Abrahams MS, Dobbs MB, Luhmann SJ, Schoenecker PL. Early reduction, arthrotomy, and cannulated screw fixation in unstable slipped capital femoral epiphysis treatment. $J$ Pediatr Orthop. 2002;22:352-358.

7. Hanley JA, McNeil BJ. The meaning and use of the area under a receiver operating characteristic (ROC) curve. Radiology. 1982;143:29-36.

8. Herrera-Soto JA, Duffy MF, Birnbaum MA, Vander Have KL. Increased intracapsular pressures after unstable slipped capital femoral epiphysis. J Pediatr Orthop. 2008;28:723-728.

9. Huber H, Dora C, Ramseier LE, Buck F, Dierauer S. Adolescent slipped capital femoral epiphysis treated by a modified Dunn osteotomy with surgical hip dislocation. J Bone Joint Surg Br. 2011;93:833-838.

10. Ibrahim T, Mahmoud S, Riaz M, Hegazy A, Little DG. Hip decompression of unstable slipped capital femoral epiphysis: a systematic review and meta-analysis. $J$ Child Orthop. 2015;9:113-120.

11. Krahn TH, Canale ST, Beaty JH, Warner WC, Lourenco P. Longterm follow-up of patients with avascular necrosis after treatment of slipped capital femoral epiphysis. $J$ Pediatr Orthop. 1993;13:154-158.

12. Larson AN, McIntosh AL, Trousdale RT, Lewallen DG. Avascular necrosis most common indication for hip arthroplasty in patients with slipped capital femoral epiphysis. J Pediatr Orthop. 2010;30:767-773.

13. Leunig M, Slongo T, Ganz R. Subcapital realignment in slipped capital femoral epiphysis: surgical hip dislocation and trimming of the stable trochanter to protect the perfusion of the epiphysis. Instr Course Lect. 2008;57:499-507.

14. Leunig M, Slongo T, Kleinschmidt M, Ganz R. Subcapital correction osteotomy in slipped capital femoral epiphysis by means of surgical hip dislocation. Oper Orthop Traumatol. 2007;19:389-410.

15. Loder RT. What is the cause of avascular necrosis in unstable slipped capital femoral epiphysis and what can be done to lower the rate? J Pediatr Orthop. 2013;33(Suppl 1):S88-91.

16. Loder RT, Richards BS, Shapiro PS, Reznick LR, Aronson DD. Acute slipped capital femoral epiphysis: the importance of physeal stability. J Bone Joint Surg Am. 1993;75:1134-1140.

17. Lowndes S, Khanna A, Emery D, Sim J, Maffulli N. Management of unstable slipped upper femoral epiphysis: a meta-analysis. $\mathrm{Br}$ Med Bull. 2009;90:133-146.

18. Madan SS, Cooper AP, Davies AG. The treament of severe slipped capital femoral epiphysis via the Ganz surgical dislocation approach-a prospective study. Bone Joint J 2013;95:424429. 
19. Palocaren T, Holmes L, Rogers K, Kumar SJ. Outcome of in situ pinning in patients with unstable slipped capital femoral epiphysis: assessment of risk factors associated with avascular necrosis. J Pediatr Orthop. 2010;30:31-36.

20. Parsch K, Weller S, Parsch D. Open reduction and smooth Kirschner wire fixation for unstable slipped capital femoral epiphysis. J Pediatr Orthop. 2009;29:1-8.

21. Ramachandran M, Ward K, Brown RR, Munns CF, Cowell CT, Little DG. Intravenous bisphosphonate therapy for traumatic osteonecrosis of the femoral head in adolescents. $J$ Bone Joint Surg Am. 2007;89:1727-1734.

22. Rosner B. Fundamentals of Biostatistics. Boston, MA, USA: Brooks/Cole; 2011.

23. Sankar WN, McPartland TG, Millis MB, Kim YJ. The unstable slipped capital femoral epiphysis: risk factors for osteonecrosis. J Pediatr Orthop. 2010;30:544-548.

24. Sankar WN, Vanderhave KL, Matheney T, Herrera-Soto JA, Karlen JW. The modified Dunn procedure for unstable slipped capital femoral epiphysis: a multicenter perspective. J Bone Joint Surg Am. 2013;95:585-591.

25. Souder CD, Bomar JD, Wenger DR. The role of capital realignment versus in situ stabilization for the treatment of slipped capital femoral epiphysis. J Pediatr Orthop. 2014;34:791-798.

26. Southwick WO. Osteotomy through the lesser trochanter for slipped capital femoral epiphysis. J Bone Joint Surg Am. 1967;49:807-835.

27. Tokmakova KP, Stanton RP, Mason DE. Factors influencing the development of osteonecrosis in patients treated for slipped capital femoral epiphysis. J Bone Joint Surg Am. 2003;85:798-801.

28. Tosounidis T, Stengel D, Kontakis G, Scott B, Templeton P, Giannoudis PV. Prognostic significance of stability in slipped upper femoral epiphysis: a systematic review and meta-analysis. J Pediatr. 2010;157:674-680, 680 e671.

29. Zaltz I, Baca G, Clohisy JC. Unstable SCFE: review of treatment modalities and prevalence of osteonecrosis. Clin Orthop Relat Res. 2013;471:2192-2198.

30. Ziebarth K, Leunig M, Slongo T, Kim YJ, Ganz R. Slipped capital femoral epiphysis: relevant pathophysiological findings with open surgery. Clin Orthop Relat Res. 2013;471:2156-2162.

31. Ziebarth K, Zilkens C, Spencer S, Leunig M, Ganz R, Kim YJ. Capital realignment for moderate and severe SCFE using a modified Dunn procedure. Clin Orthop Relat Res. 2009;467:704716. 